\title{
A rare case of giant fibrolipoma of the thigh in a child
}

\author{
Andreea Oltean ${ }^{1}$, Cristina Oana Marginean ${ }^{2}$, Horea Gozar ${ }^{3}$, Horvath Emoke ${ }^{4}$, \\ Mihaela-Ioana Chincesan ${ }^{2}$ \\ ${ }^{1}$ Pediatrics Clinic I, Emergency County Hospital Targu Mures, Romania \\ 2Pediatrics Discipline, University of Medicine and Pharmacy Targu Mures, Romania \\ ${ }^{3}$ Department of Pediatric Surgery and Orthopedics, University of Medicine and Pharmacy, \\ Targu Mures, Romania \\ ${ }^{4}$ Pathological Anatomy Discipline, University of Medicine and Pharmacy Targu Mures, Romania
}

\begin{abstract}
Lipomas are benign tumors consisting of fat tissue that are typically located just beneath the skin, they are most often found on the upper back, shoulders, arms and buttocks. Less commonly, they can be found in deeper tissue of the thigh. Most lipomas are small, weigh only a few grams, and the maximum diameter is under 2 $\mathrm{cm}$, but "giant" lipomas have at least $10 \mathrm{~cm}$ in diameter and a minimum of 1,000 g. We present the case of a 14 years old female patient that noticed for about two and a half months, the appearance of a tumor formation in the front side of the left thigh. She was hospitalized for investigations in the department of Pediatric Hematolo-gy-Oncology Targu Mures, of Pediatric Clinic I, under the suspicion of soft tissue tumor. The magnetic resonance (MRI) performed revealed a left thigh intramuscular lipoma with maximum dimensions of $66 / 44 / 125 \mathrm{~mm}$, later being transferred to the Clinic for Pediatric Surgery and Orthopedics for removal. The histopathologic exam revealed fibrolipoma.
\end{abstract}

Keywords: lipoma, thigh, child

\begin{abstract}
Abbreviations
$\mathbf{E S R}=$ erythrocyte sedimentation rate

$\mathbf{C R P}=\mathrm{C}$ reactive protein
\end{abstract}

\section{INTRODUCTION}

Lipomas are accumulations of adipose tissue that occur more frequently at the back of the neck, on the trunk and proximal limbs but they may develop in virtually all organs throughout the body being localized intraabdominally, intramuscularly or subcutaneously. They occur in $1 \%$ of the population, children being rarely affected. These slowgrowing, benign fatty tumors form soft, lobulated masses that are enclosed by a thin, fibrous capsule. They are compressible and can suffer a series of transformations such as: atrophy, calcification, liquefaction, or xantomatosis, that can complicate their evolution (1). Their volume tends to increase with the increase in body weight, but it does not regress as a consequence of weight loss. Multiple lipomas, identical or isolated lipomas can be autosomal dominant transmitted and usually appear in the third decade of life in patients with familial multiple lipomatosis. Speculation exists regarding a potential link between trauma and subsequent lipoma formation $(1,2)$.

The differential diagnosis is provided by computed tomography (CT), magnetic resonance imaging (MRI), biopsy and/or immunohistochemistry (3). The most common malignant soft tissue mass is malignant fibrous histiocytoma followed by liposarcoma, which resembles lipomas on computed tomography and MRI and is most common in the thigh (4). Magnetic resonance imaging with contrast depicts the lipomatous mass as having a char- 
acteristic high-intensity fat signal without enhancement (5).

Usually, treatment of a lipoma is not necessary, unless the tumor becomes painful or restricts movement. They are usually removed for cosmetic reasons, if they grow very large or for histopathology when there is a suspicion of liposarcoma (6). Rapidly growing or painful lipomas can be treated with a variety of procedures ranging from steroid injections to excision of the tumor. The outcome and prognosis are excellent for benign lipomas. Recurrence is uncommon but may develop if the excision is incomplete.

Their possible malignant character and their tendencies of reccurence after removal are the reasons why we want to focus on them.

\section{CASE REPORT}

We present the case of a 14 years old, female patient, without pathological personal history, who noticed - for about two and a half months - the appearance of a tumor formation on the front side of the left thigh rapidly increasing in size, without other symptoms. She presented for an ambulatory pediatric consultation, from where she was admitted at the Hematology-Oncology Department of Pediatrics for investigations, under the suspicion of a soft tissue tumor. Clinical examination at the presentation revealed: a general good state, normal skin color, bilateral thigh stretch marks, without palpable lymph nodes or organomegaly, cardiac and respiratory evaluation was normal, musculoskeletal examination revealed a palpable mobile mass on the front side of the left thigh, with minimal tenderness, firm in consistency, with approximate dimensions of $13 / 7 / 8 \mathrm{~cm}$, without motor deficit (Fig. 1 a,b). Actual body weight was $62 \mathrm{~kg}$ (from the anamnesis it shows that the body weight before the appearance of the tumor was about $60 \mathrm{~kg}$ ). There was no history of trauma or fever.

The patient was investigated paraclinically and imagistically. Laboratory tests revealed normal values of non-specific tumoral markers such as ferritine, serum copper, alkaline phosphatase, serum calcium, lactate dehydrogenase, absent inflammatory syndrome (ESR, CRP normal), normal coagulation panel and creatin-phosphokinase, normal blood count and peripheral blood smear. Serum electrolytes, blood glucose, renal, liver function tests and electrocardiogram were normal. Ultrasound of the thigh described: a well-defined elliptical area in the vast medial muscle and distal medial, with deviation of the vascular structures form proximity, with $<12 / 5.5 / 7.4 \mathrm{~cm}$, with a homogeneous structure that mimics the structure of muscle tissue without Doppler vascular signal or vascular calcifications at this level.

Abdomen ultrasound and pulmonary x-ray performed revealed no abnormality. Diagnostic investigations were completed with MR that revealed: deep intramuscular lipoma located above the middle third of the left thigh, in the quadriceps muscle - the vast medial, posterior coming into contact
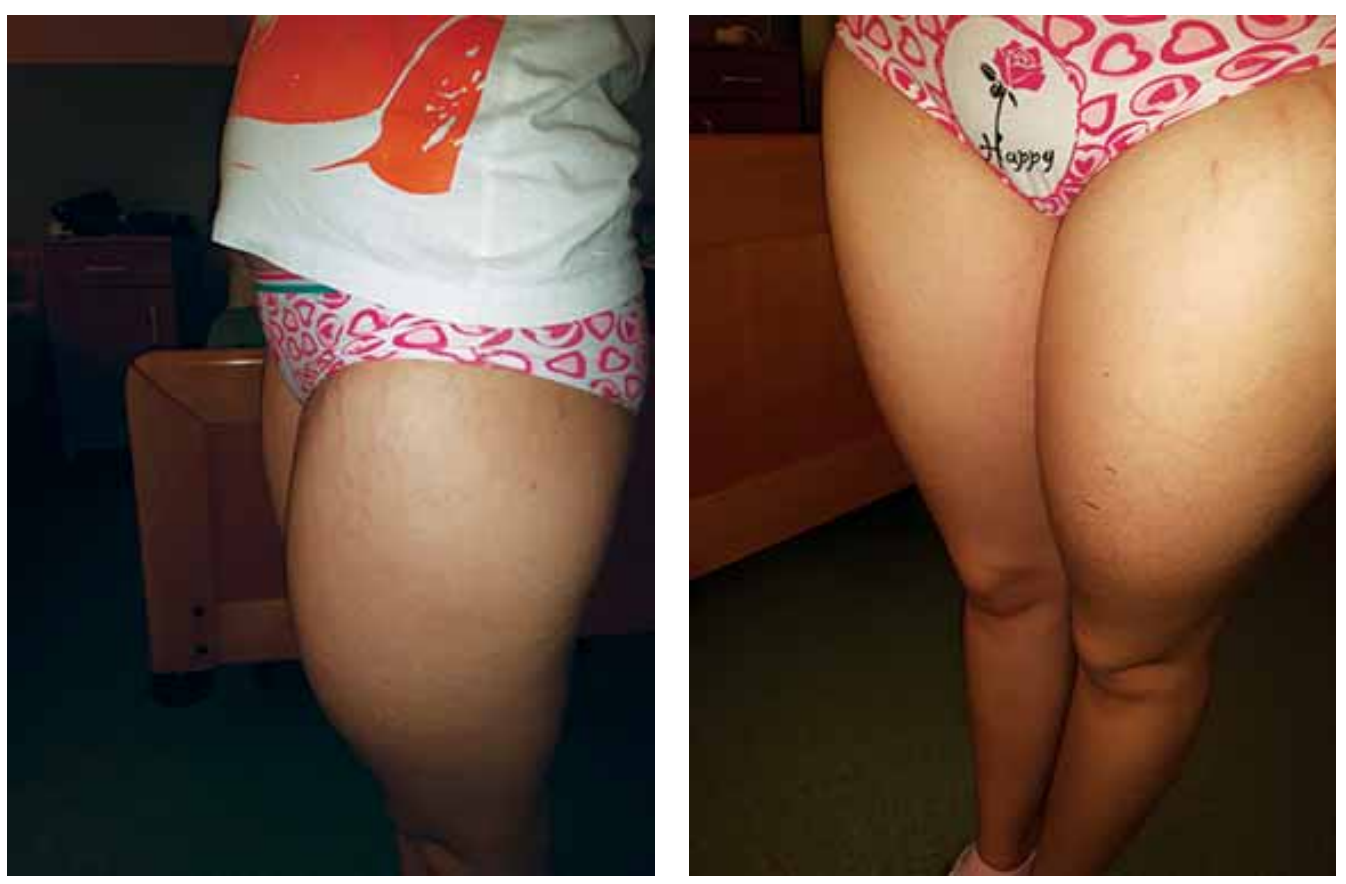

FIGURE 1a and $\mathbf{1 b}$. Tumor formation on the front of the left thigh, stretch marks 


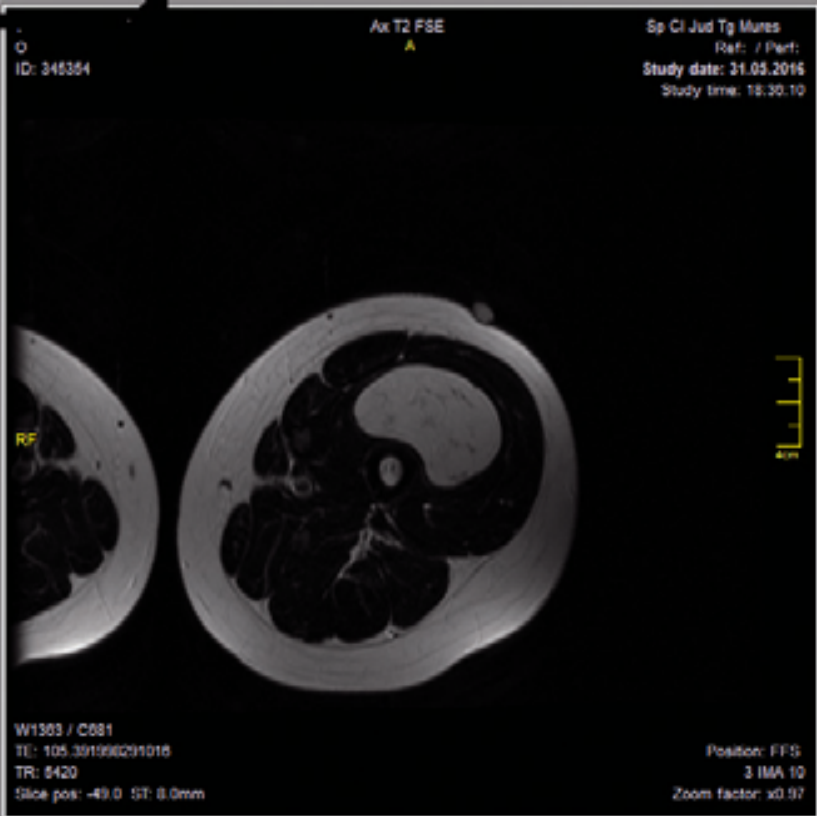

FIGURE 2a. Axial section in the vast medial muscle - a well-defined, homogeneous formation, with high-intensity signal in $\mathrm{T} 2$ is observed.

with the femoral shaft, well defined, with fine septa, with $>66 / 44 / 125 \mathrm{~mm}$, without compression on the femoral vascular package (Fig. 2 a,b).

The patient was transferred to the Pediatric Surgery Clinic for the complete excision of the lipoma and histological examination. The macroscopic description revealed that the tumour was adipose tissue, it was capsulated, lobulated, of elastic consistency and the diameter was $130 / 60 / 50 \mathrm{~mm}$, weight of $960 \mathrm{~g}$. Microscopic description revealed a tumour consisting of numerous adipose panicles, mature cells without atypia, separated by fibrous tissue strips. Histiocytes are observed around fibrous septa (Fig. 3 a,b). Cellular protein S100 is negative and the

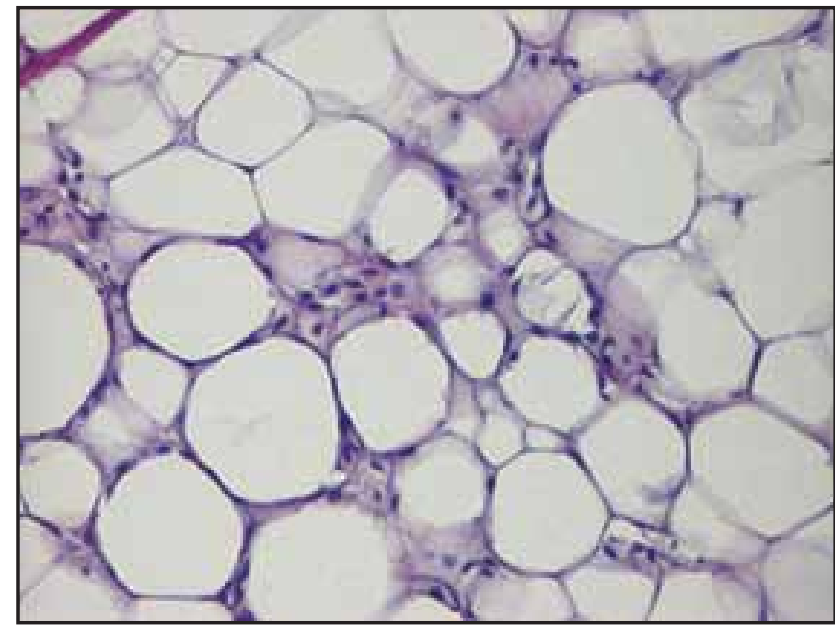

FIGURE 3a. Mature adipose cell aggregates without atypia (hematoxylin-eosin stain, 20 x magnification)

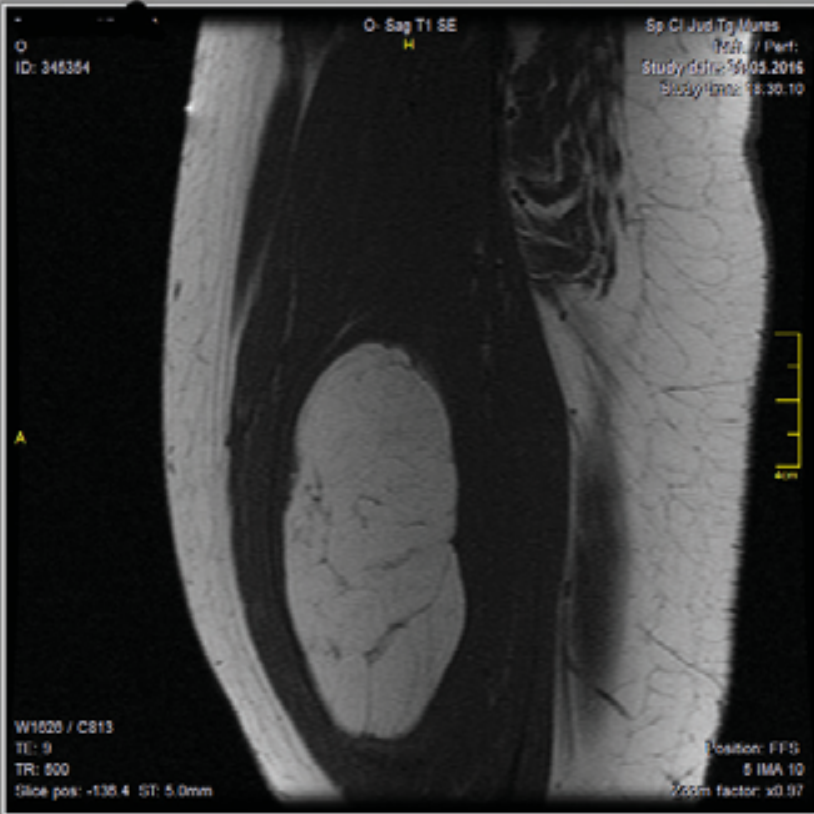

FIGURE 2b. Sagittal section in the vast medial muscle - a homogeneous oval formation, well defined, with fine septa included, with high-intensity signal in T1 is observed.

$\mathrm{Ki}-67$ proliferation index is low, the presence of a predominantly lymphocytic inflammatory infiltrate was also observed. Conclusion of the histologic examination was fibrolipoma completely excised.

\section{DISCUSSIONS}

Lipomas are frequent superficial or deep, benign soft tissue tumors, clinically appearing as asymptomatic lesions but in some cases they can reach a considerable size (7). In the presented case the lipoma was asymptomatic, and its giant volume was associated with the increasing body weight of the patient, also visible through the tights striae.

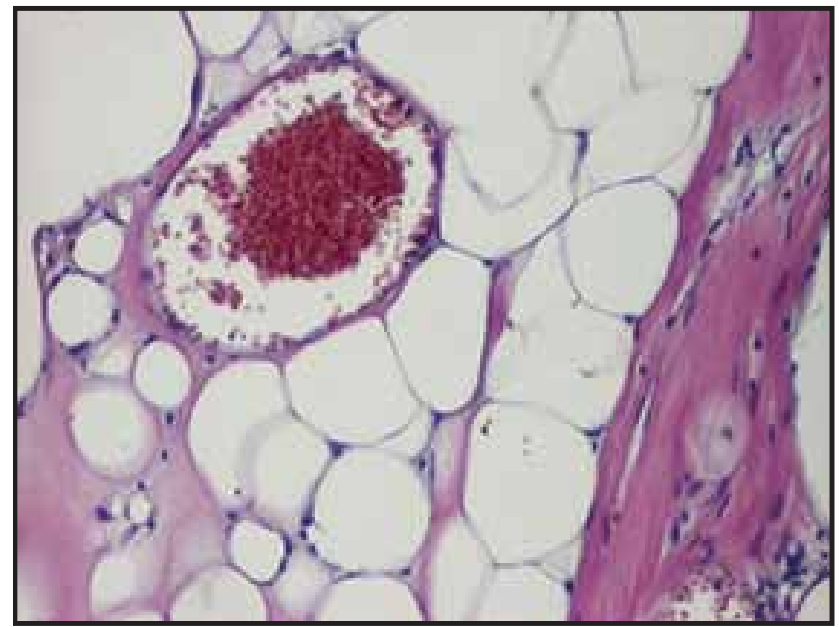

FIGURE 3b. Fibrous tissue and histiocytes (hematoxylineosin stain, 20 x magnification) 
Terzioglu et al. describe lipomas of 10.0/5.0/3.0 $\mathrm{cm}$ or greater as giant lipomas and note that tumors of this size should include liposarcomas in the differential diagnosis (8). In the presented case, the MRI measurements of the lipoma indicated large dimensions $(6.6 / 4.4 / 12.5 \mathrm{~cm})$, this helped both framing the giant lipoma and also for differential diagnosis.

Ultrasound is the diagnostic imaging method of choice due to its availability and low cost when compared with MRI, in the initial assessment of soft tissue lesions whose origin and nature are not fully understood (9). In the present case, ultrasound did not permit exact definition of the lesion and Doppler showed complete absence of signal. Despite the fact that the ultrasound could not differentiate between a benign lesion and a lesion of lowgrade malignancy, diagnosis of giant lipoma was completed with MRI which was confirmed by subsequent histological examination.

Because of its excellent tissue contrast and lack of ionizing radiation, magnetic resonance imaging has become the modality of choice in the evaluation of deep and large soft-tissue masses in children, defining the extent of the lipoma and its relationship to adjacent structures, and performing post-therapy follow-up (10).

Meticulous assessment of the margins and internal characteristics on MRI can be a useful aid in further distinguishing between lipoma and welldifferentiated liposarcoma $(10,11)$. MRI examination in the case presented showed not only the dimensions of the lipoma but also the fact that it was deep intramuscular, well defined, with fine septa and without compression on the femoral vascular package.

Bjerregaard et al. showed in their study it is essential to confirm the diagnosis of lipoma by excisional biopsy, although preoperative imaging with CT scan is useful (12). Histological examination in case presented revealed it was a giant fibrolipoma completely excised.
Serpell at al. have concluded that the indications for excision of a lipoma include a mass greater than $5 \mathrm{~cm}$ in size, a subfascial location, a tumor that is growing, clinical features such as pain, firmness or irregularity, or a questionable preoperative needle biopsy result but also the patient's cosmetic concern (13). Small lipomas can be removed by enucleation. Large lipomas are removed by performing an incision in the skin covering the lipoma (14). Treatment of the lipoma in this case was complete excision because of the rapidly increasing size, cosmetic concern and also histological examination in order to clarify the positive diagnosis.

Fibrolipomas are extremely rare in pediatric patients. Literature describes a case of congenital fibrolipoma of the lower left limb completely excised in a 4 year old patient (15). However there is a risk of recurrence after a variable period despite radical resection. This may occur as malignant transformation.

\section{CONCLUSIONS}

Lipomas are benign soft tissue masses and should always be considered in the differential diagnosis of a thigh mass. It is important to recognize the clinical signs of a superficial or deep lipoma and the imaging manifestations of a soft tissue lipoma to optimize an initial diagnosis and treatment. Ultrasonography is used for the initial assessment for small, superficial lesions or when the clinical history strongly suggests a specific diagnosis, however in the case of giant lipoma it can not differentiate between a benign or malign lesion. Magnetic resonance imaging is frequently required in the evaluation of lipomas, along with histological examination to confirm the diagnosis, particularly for those that are deep or large and those in which ultrasound have been inaccurate.

Treatment of the lipoma involves complete excision but recurrence may be possible, so long-term follow-up of these patients may be necessary as a precautionary measure.

\section{REFERENCES}

1. Kliegman R.M. Tumors of the skin. In: Behrman R.E, Jenson H.B, Stanton B.F. Nelson Textbook of Pediatrics, 18th ed, Saunders Elsevier 2007, 669: 2767

2. Signorini M., Campiglio G.L. Posttraumatic lipomas: where do they really come from? Plast Reconstr Surg 1998; 101(3):699-705.

3. Grivas T.B., Psarakis S.A., Kaspiris A. et al. Giant lipoma of the thenar - case study and contemporary approach to its aetiopathogenicity. Hand (N Y) 2009; 4(2):173-176.

4. Murphey M.D., Carroll J.F., Flemming D.J. et al. From the archives of the AFIP: benign musculoskeletal lipomatous lesions. RadioGraphics. 2004; 24(5):1433-1466.

5. Kransdorf M.J., Bancroft L.W., Peterson J.J. et al. Imaging of fatty tumors: distinction of lipoma and well-differentiated liposarcoma. Radiol 2002; 224(1):99-104.

6. Salam G.A. Lipoma excision. Am Fam Physician 2002; 65 (5): 901-904.

7. Whittle C., Cortés M., Baldassare G. et al. Subgaleal lipomas: ultrasound findings. Rev Med Chil 2008; 136(3):334-337.

8. Terzioglu A., Tuncali D., Yuksel A. et al. Giant lipomas: a series of 12 consecutive cases and a giant liposarcoma of the thigh. Dermatol Surg 2004; 30(3):463-467. 
9. Widmann G., Riedl A., Schoepf D. et al. State-of-the-art HR-US imaging findings of the most frequent musculoskeletal soft-tissue tumors. Skeletal Radiol 2009;38(7):637-649.

10. Oscar M. Navarro, Eoghan E.L., Bo-Yee N. Pediatric Soft-Tissue Tumors and Pseudotumors: MR Imaging Features with Pathologic Correlation Part 1. Imaging Approach, Pseudotumors, Vascular Lesions, and Adipocytic Tumors, RadioGraphics 2009; 29:887-906.

11. Ohguri T., Aoki T., Hisaoka M. et al. Differential diagnosis of benign peripheral lipoma from well-differentiated liposarcoma on MR imaging: is comparison of margins and internal characteristics useful? $\mathrm{Am} \mathrm{J}$ Roentgenol 2003; 180(6):1689-1694.
12. Bjerregaard P., Hagen K., Daugaard S. et al. Intramuscular lipoma of the lower limb. Long-term follow-up after local resection. J Bone Joint Surg Br 1989; 71(5): 812-815.

13. Serpell J.W., Chen R.Y. Review of large deep lipomatous tumours. ANZ J Surg. 2007; 77(7):524-529.

14. William J.D. Lipomas In: Berger T.G.; Elston D.M. Andrews' Diseases of the Skin: Clinical Dermatology 10th ed. London Elsevier 2005; 780-781.

15. Nilüfer A., Umut H.G., Hakan A. et al. Giant Fibrolipoma Extending Throughout a Whole Extremity: A Rare Child Case Report, J Clin Anal Med 2016;7(2): 174-176

Conflict of interest: none declared Financial support: none declared 